\title{
Características estruturais maculares de olhos de pré-escolares nascidos prematuros: análise por tomografia de coerência óptica e oftalmoscopia binocular indireta
}

\author{
Structural features of macular eyes of preschoolers born preterm: analysis by optical coherence \\ tomography, and indirect ophthalmoscopy
}

Lígia Beatriz Bonottoํ․ Ana Tereza Ramos Moreira², Cristina Martins Faria Bortolotto ${ }^{3}$

\section{RESUMO}

Objetivo: Comparar a estrutura retiniana da mácula e fóvea entre prematuros com retinopatia da prematuridade (ROP) estágios II e III pós-tratamento, com ROP estágios II e III regredida espontaneamente e sem ROP, através de exames de tomografia de coerência óptica (OCT) e da oftalmoscopia binocular indireta (OBI).

Métodos: Estudo do tipo transversal, observacional e não cego. Foram incluídas criancas prematuras nascidas entre 06/1992 e6/2006 examinadas entre 06/2009 e 12/2010; idade gestacional menor ou igual a 32 semanas e peso ao nascer menor ou igual a 1.599 g;com mínimo de três consultas durante o período de seleção; sem retinopatia da prematuridade ou com diagnóstico de ROP estágios II ou III em pelo menos um dos olhos com regressão espontânea ou após tratamento; máximo de seis meses de idade cronológica para o primeiro exame no serviço; idade cronológica mínima de quatro anos no período da reavaliação. Foram excluídas crianças prematuras que não compareceram ou que não tinham condições clínicas para a realização dos exames de reavaliação. Os prematuros foram divididos em três grupos: G1- com ROP pós-tratamento; G2- com ROP pós-regressão espontânea; e G3- sem ROP. Os exames realizados foram OBI e OCT.

Resultados: Vinte e quatro prematuros (48 olhos) apresentaram os critérios exigidos para a pesquisa, com idade média cronológica entre 5 e 6 anos. À OBI, houve diferença estatística significativa para a presença de alterações na retina dos prematuros do grupo G1. No entanto estas alterações corresponderam às lesões cicatriciais deixadas pelo tratamento da ROP, sem comprometimento visível da região macular. À OCT houve diferença estatística significativa para a maior espessura foveal para os prematuros do grupo G1. Considerando-se o olho esquerdo, não houve diferença estatística significativa relacionada à espessura da fóvea entre G1 e G3. Não houve diferença entre os três grupos estudados quanto às alterações encontradas nas camadas da retina ao OCT.

Conclusão: Os prematuros com ROP pós-tratamento apresentaram espessura foveal maior que os prematuros com ROP pós-regressão espontânea e espessura foveal semelhante aos prematuros sem ROP em relação à avaliação do olho esquerdo. Em relação às alterações das camadas da retina detectadas ao OCT, os três grupos foram semelhantes, sem expressão de diferença para o grupo tratado neste estudo.

Descritores: Retinopatia da prematuridade; Mácula lutea/anatomia \& histologia; Tomografia de coerência óptica; Oftalmoscopia; Pré-escolar

\begin{abstract}
Purpose: Compare the retinal structure of the macula and fovea among premature infants with retinopathy of prematurity (ROP) stages II and III post treatment, premature infants with ROP stages II and III with spontaneous regression and premature infants without ROP, through optical coherence tomography (OCT) and binocular indirect ophthalmoscopy (BIO) examinations.

Methods: Cross-sectional observational and not-blinded study. There were included premature infants born between 06/1992 and 06/2006 and examined between 06/2009 and 12/2010; gestational age less than or equal to 32 weeks and birth weight less than or equal to 1,599 g: with a minimum of three visits during the selection period; without retinopathy of prematurity, or with the diagnosis of ROP stages II or III in at least one eye with spontaneous regression or after ROP treatment; maximum of six months of chronological age for the firstexamination at the service; minimal chronological age of four years old in the reassessment period. There were excluded premature infants who did not attend or did not have clinical conditions for the reassessment examination. The premature infants were divided into three groups: G1 - with ROP post-treatment; G2 - with ROP post-spontaneous regression; and G3 - without ROP. The exams performed were $B I O$ and OCT.
\end{abstract}

Results: Twenty-four premature infants (48 eyes) presented the criteria required for the research, chronological age ranging from 5 to 6 years. At BIO, there was a statistically significant difference for the presence of alterations in the retina of premature infants from group G1. However these changes corresponded to the cicatricial lesions left by the ROP treatment, without visible impairment to the macular region. At OCT there were statistically significant differences for the greatest foveal thickness between premature infants from groups G1 and G2. Considering the left eye, there was no statistically significant difference related to the thickness of the fovea between G1 and G3. There was no difference among the three groups studied in relation to the changes of the retinal layers at OCT.

Conclusion: Premature infants with ROP post-treatment showed foveal thickness greater than premature infants with ROP post-spontaneous regression; and foveal thickness similar to premature infants without $R O P$ in relation to assessment of the left eye. Regarding the changes of the retinal layers detected at OCT, the three groups were similar, without expression of difference for the treated group in this study.

Keywords: Retinopathy of prematurity; Macula lutea/anatomy \& histology; Tomography, optical coherence; Ophthalmoscopy; Child, preschool

\section{INTRODUÇÃO}

O estudo da interferometria óptica iniciou-se em 1969 e deu origem aos equipamentos atuais de tomografia de coerência óptica $(\mathrm{OCT})^{(1)}$. A partir dos anos 90 com contínua progressão, houve grande impulso no conhecimento e na aplicabilidade médica deste método que utiliza uma imagem refletida da luz do laser após atravessar as camadas do tecido, in vivo(2).

Atualmente os aparelhos de tomografia de coerência óptica (OCT) apresentam dados comparativos de normalidade padronizados para comparação entre adultos acima de 18 anos. Abaixo desta
Financiamento: Não houve financiamento para este trabalho.

Divulgação de potenciais conflitos de interesse: L.B.Bonotto, None; A.T.R.Moreira, None C.M.F.Bortolotto, None

Endereço para correspondência: Lígia Beatriz Bonotto. Rua Abdon Batista, 146 - Joinville (SC) 89201-010 - Brasil

E-mail: ligia@oftalmopediatria.com.br / ligiaoftalmopediatria@sadalla.com.br 
idade, não existe ainda uma escala normativa de valores. Vários fatores podem interferir nas medidas do nervo óptico e da espessura foveal e macular como: idade, peso ao nascimento, raça, refração e ambliopia. A busca de uma padronização para as medidas retinianas ao OCT entre a população infantil deu origem a vários trabalhos. Nestes estudos, a espessura foveal variou de 157 a 221 micra entre crianças consideradas saudáveis do ponto de vista oftalmológico e clínico geral, na idade variável entre 1 e 18 anos. Estas medidas podem servir de base comparativa para o presente estudo ${ }^{(3-5)}$.

Entre as crianças nascidas prematuramente observam-se, ao exame de OCT, alterações típicas na região macular e foveal quando comparadas às crianças nascidas a termo ${ }^{(6-8)}$. A ciência ainda não tem uma resposta precisa para explicar o que realmente acontece com a maturação destas camadas retinianas, entre nascidos prematuros ${ }^{(6)}$.

Em 2010, nos EUA, pesquisadores compararam a avaliação das imagens por OCT de pacientes adultos nascidos prematuros, e adultos nascidos a termo. Os autores observaram uma alteração na arquitetura foveal dos pacientes com história de prematuridade, mas a maioria deles apresentava boa acuidade visual( ${ }^{(7)}$.

No Brasil, o único estudo publicado sobre a estruturação retiniana ao OCT foi conduzido entre 12 prematuros, todos portadores de retinopatia da prematuridade (ROP), e idade variável entre 45 e 90 dias de vida. Nestes, a OCT demonstrou camadas retinianas pouco diferenciadas com aumento da refletividade do epitélio da retina pigmentar-coriocapilar na área macular ${ }^{(8)}$.

Em vários estudos, observa-se que a aparência oftalmoscópica da retina, especialmente mácula e fóvea, não está diretamente ligada à resposta visual. Olhos em que retina apresenta repuxamento do feixe papilo-macular ("draping") e ausência de depressão foveal podem desenvolver visão normal ou próxima ao normal. Os estudos do aspecto retinográfico e ao OCT não esclarecem como olhos com retina aparentemente normal podem apresentar um contorno anormal ao OCT (9-11).

Atualmente a OCT é considerada como uma ferramenta para meIhor compreender as particularidades do desenvolvimento maculare retiniano dos prematuros, tanto na fase ativa da ROP como após esta fase. No entanto, a OBI ainda é considerada como a melhor maneira de se avaliar as retinas dos prematuros. A OCT realizada na fase ativa da ROP, apresentou vantagem em comparação à OBI ao mostrar alterações pré-retinianas e retinianas (cistos maculares e membranas pré-retinianas) que classificariam a ROP em estágios mais avançados que o exame padrão ouro (OBI). No entanto, para as alterações vasculares, a OBI foi superior a OCT de controle portátil(12).

Com a intenção de se avaliar se os pré-escolares de nascimento prematuro e com retinopatia regredida após tratamento apresentavam maior incidência de alteração macular e foveal do que os prematuros com retinopatia de regressão espontânea, este estudo teve como objetivo comparar a estrutura retiniana da mácula e da fóvea entre prematuros com ROP estágios II e III pós-tratamento, prematuros com ROP estágios II e III regredida espontaneamente e prematuros sem ROP, através de exames de OCT e da oftalmoscopia binocular indireta $(\mathrm{OBI})$.

\section{MÉTODOS}

Estudo transversal observacional não cego.

Critérios de inclusão: crianças pré-escolares que tiveram nascimento prematuro durante o período de junho de 1992 e junho de 2006; idade gestacional ( $\mathrm{G}$ ) $\leq 32$ semanas e peso ao nascimento (PN) $\leq 1.599 \mathrm{~g}$; que tiveram realizado o mínimo de três consultas durante o período de seleção; com diagnóstico de ROP estágios II ou III em pelo menos um dos olhos com regressão espontânea ou terem sido submetido a tratamento da ROP; terem realizado o primeiro exame no serviço até seis meses de idade cronológica; e possuírem idade cronológica mínima de quatro anos no período da reavaliação (junho/2009 a dezembro/2010).
Critérios de exclusão: dentre os incluídos no estudo, os que não puderam comparecer ou cujas condições clínicas não permitiam comparecimento para os exames de reavaliação, como em caso de comprometimento severo do sistema nervoso central ou de síndromes impeditivas.

Os prematuros, que cumpriram com os critérios de inclusão e que obtiveram dos seus responsáveis legais o consentimento esclarecido assinado em relação aos exames requeridos pelo estudo foram divididos nos seguintes grupos:

- Grupo G1 - recém-nascidos prematuros que apresentaram ROP, estágios II e III e que foram submetidos ao tratamento por laser ou por crioterapia;

- Grupo G2 - recém-nascidos prematuros que apresentaram ROP, estágios II e III, com regressão espontânea da doença;

- Grupo G3 - recém-nascidos prematuros que não desenvolveram a ROP.

As crianças pré-escolares agrupadas nos três grupos descritos acima foram submetidas à OBI sob midríase (ciclopentolato a $1 \%$ e tropicamida a 1\%), com imagens arquivadas em DVD. A OBI foi realizada pela mesma profissional durante o período de seleção e durante a reavaliação. Na avaliação através da OBI, foram utilizadas as mesmas referências quanto à localização das alterações retinianas utilizadas na Classificação Internacional da ROP(13,14): a. normal (NL), OBI normal; b. alterado (ALTNT), alteração não causada pelo tratamento da ROP, como tração dos vasos da região macular, prega fibrosa no feixe papilo-macular ou ectopia da região macular; c. alteração (PCZ2 e 3), com pontos cicatrizados do tratamento entre as zonas II e III da retina; d. alteração ( $P C Z 1$ e 2), com pontos cicatrizados do tratamento entre as zonas I e II; e. alteração (PCZ2), com pontos de cicatrização a partir da zona II; f. DTTR, descolamento total tardio de retina. Para o cálculo da diferença estatística em relação à presença de alterações retinianas à fundoscopia (OBI) entre os grupos estudados, todos os tipos de alteração encontrados foram agrupados em único grupo (grupo com alteração).

A OCT foi realizada em todas as crianças em ambos os olhos, sob midríase máxima (tropicamida a 0,5\% e fenilefrina a 5\%, em cada olho por três vezes antes do exame), utilizando-se o equipamento Cirrus HD-OCT (Carl Zeiss Meditec Inc.). As análises foram realizadas mediante a aplicação de programas específicos para cortes tomográficos: "macular cube $512 \times 128$ " e "5 radial raster", cortes aquisição de 5 a 10 imagens para cada olho com comprimento de $6 \mathrm{~mm}$, e duração variável entre 5 e 20 minutos. Todos os exames foram realizados pelo mesmo profissional. Neste estudo, as alterações observadas nas camadas retinianas foram classificadas conforme a quantidade de alterações e camadas envolvidas: a. Normal (NL), sem alteração; b. 1 ALT, presença de uma alteração (ex.: retificação do contorno foveal); c. 2 ALT, presença de duas alterações (ex.: retificação do contorno foveal e espessamento da região macular); d. 3 ALT, presença de três alterações (ex.: contorno foveal atenuado, espessamento da região macular e aumento da refletividade da camada interna da retina).

Para os exames de OBI e OCT não foram utilizados formulários padronizados e os profissionais executantes dos exames tiveram acesso aos resultados.

Para o cálculo de diferença estatística entre os grupos estudados, todas as alterações foram agrupadas em único grupo, indiferentemente ao número de alterações ou às camadas envolvidas.

Os resultados da OCT foram avaliados comparando-se o grupo de pacientes tratados com os que apresentaram regressão espontânea da retinopatia. E posteriormente este grupo foi comparado com os prematuros sem ROP, para avaliar o significado da agressividade da ROP nas possíveis alterações encontradas.

\section{Análise estatística}

Para avaliação da associação entre variáveis dicotômicas, foi considerado o teste Exato de Fisher (exemplo: $\mathrm{OBI}$ alterado versus OBI 
normal; OCT de retina alterada versus OCT normal). Para a comparação entre grupos em relação às variáveis quantitativas, foi considerado o teste não paramétrico de Mann-Whitney. Os valores de $p<0,05$ indicaram significância estatística para ambos os testes utilizados no estudo, considerando-se o intervalo de confiança de 95\%. O estudo foi realizado para cada olho direito e para cada olho esquerdo, de cada prematuro, dos três grupos estudados. E a comparação entre os grupos foi realizada, separadamente, para os olhos direitos e para os olhos esquerdos de cada grupo. Os dados foram analisados com o programa computacional Statistica v.8.0.

O estudo foi avaliado e aprovado pelo Comitê de Ética em Pesquisa do Hospital São José, Joinville, SC, onde o HOSAG está vinculado.

\section{RESULTADOS}

Foram avaliados para o estudo 432 recém-nascidos prematuros cadastrados e examinados para ROP no período de seleção (junho de 1992 a junho de 2006), no referido Hospital de Olhos. Destes, 266 não foram selecionados para reavaliação, por apresentarem idade gestacional e peso ao nascimento maior que o recomendado nos critérios de inclusão.

Para a reavaliação proposta na idade pré-escolar, permaneceram 166 prematuros deste grupo avaliado. Destes, 103 foram excluídos por não cumprirem com os outros critérios de inclusão; 39 foram excluídos por dificuldade de contato, óbito, acompanhamento em outro serviço, entre outros motivos, como a recusa em participar ou o não comparecimento aos exames.

Para a atual pesquisa de reavaliação durante a idade pré-escolar foram incluídos 24 recém-nascidos prematuros, distribuídos nos três grupos: G1 - ROP e tratamento, 7 pacientes; G2 - ROP e regressão espontânea, 8 pacientes; e G3 - prematuros sem ROP, 9 pacientes (Quadro 1).

\section{Oftalmoscopia binocular INDIRETA}

A distribuição dos pacientes dos grupos G1 e G2, conforme a oftalmoscopia indireta da retina encontra-se resumida no quadro 2.

Todos os prematuros do grupo G3, sem ROP, apresentaram retina aplicada sem alteração no aspecto anatômico à oftalmoscopia binocular indireta.

\section{TOMOGRAFIA DE COERÊNCIA ÓPTICA}

Os aspectos da ultraestrutura retiniana observados no exame de OCT dos prematuros dos grupos G1, G2 e G3 estão representados no quadro 3.

O aspecto tomográfico descritivo da retina dos prematuros dos três grupos do estudo está representado no quadro 4.

Os resultados comparativos, relacionados à espessura foveal, entre o grupo G1 e grupo G2 e entre o grupo G1 e G3 estão representados na tabela 1.

Em relação à comparação entre os grupos G1 e G2 e entre G1 e G3, referentes às alterações nas camadas da retina, os resultados estão representados na tabela 2.
Os resultados da comparação dos exames da oftalmoscopia binocular indireta estão representados na tabela 3.

A comparação entre o grupo de prematuros com ROP tratada e o grupo de prematuros sem ROP não se justifica clinicamente.

\section{DISCUSSÃO}

Esta pesquisa reforça a ideia de que as alterações encontradas ao exame de OCT são uma manifestação da organização estrutural da retina do prematuro e parece não sofrer influência da gravidade da ROP ou do processo de tratamento exigido pela ROP em seu estágio mais severo ${ }^{(6-8)}$. Existe uma diferença estatisticamente significativa entre a espessura foveal do grupo G1 e do grupo G2, observada neste estudo. No entanto, quando se comparou o grupo de prematuros com ROP tratada com o grupo de prematuros sem ROP, não houve diferença significativa quando analisados os olhos esquerdos dos dois grupos. Esta diferença foi encontrada somente em relação aos olhos direitos destes grupos. Esta constatação não sustentou a hipótese de que a retina dos nascidos prematuros com ROP tratada teriam maior espessura foveal e mais alterações na estrutura macular. A assimetria da doença em relação aos olhos direito e esquerdo, no

Quadro 2. Características descritivas do exame de oftalmoscopia binocular indireta de pré-escolares nascidos prematuros componentes dos grupos G1 e G2 com retinopatia da prematuridade

\begin{tabular}{ccccc}
\hline Grupos & Paciente & Tipo de tratamento & Olho direito & Olho esquerdo \\
\hline G1 & 1 & Laser & PCZ 2 e 3 & PCZ 2 e 3 \\
& 2 & Laser & PCZ 2 e 3 & PCZ 2 e 3 \\
& 3 & Laser & PCZ 1 e 2** & PCZ 1 e 2 \\
& 4 & Laser & PCZ 2*** & PCZ 2 \\
5 & Laser & PCZ 2 & PCZ 2 \\
& 6 & Crioterapia & PCZ 2 & DTTR**** \\
& 7 & Laser & PCZ 2 e 3 & PCZ 2 e 3 \\
& 8 & ROP regredida & Normal & Normal \\
& 9 & ROP regredida & Normal & Normal \\
& 10 & ROP regredida & Normal & Normal \\
& 11 & ROP regredida & Normal & Normal \\
& 12 & ROP regredida & Normal & Normal \\
& 13 & ROP regredida & Normal & Normal \\
& 14 & ROP regredida & Alterado & Alterado \\
& 15 & ROP regredida & Alterado & Alterado \\
\hline
\end{tabular}

G1=ROP estágio II e III pós-tratamento; G2= ROP estágio II e III regredida; PCZ 2 e 3 e 1 e $2=$ pontos cicatriciais entre zonas 2 e 3 ; e entre zonas 1 e 2: PCZ $2=$ pontos cicatriciais a partir dazona 2; DTTR= descolamento total de retina; $\mathrm{ROP}=$ retinopatia da prematuridade.

Quadro 1. Dados demográficos dos três grupos de pré-escolares de nascimento prematuro classificados em três grupos de retinopatia da prematuridade (G1, G2, G3) considerando-se as variáveis sexo, idade cronológica na reavaliação, idade gestacional, peso ao nascimento e número de consultas entre o período de seleção e a reavaliação

\begin{tabular}{|c|c|c|c|c|c|c|c|c|c|c|c|}
\hline \multicolumn{2}{|c|}{ Grupos } & \multicolumn{2}{|c|}{ Sexo } & \multicolumn{2}{|c|}{ ICR (anos) } & \multicolumn{2}{|c|}{ IG (semanas) } & \multicolumn{2}{|c|}{ PN (gramas) } & \multicolumn{2}{|c|}{ Número de consultas } \\
\hline & N & $M(\%)$ & $F(\%)$ & Média & DP & Média & DP & Média & DP & Média & DP \\
\hline G 1 & 7 & 71 & 29 & 6,1 & 1,6 & 28,1 & 1,9 & $1.015,0$ & 123,5 & 24,4 & 20,5 \\
\hline G 2 & 8 & 25 & 75 & 6,5 & 1,9 & 27,2 & 1,6 & 963,7 & 123,0 & 19,8 & 13,9 \\
\hline G 3 & 9 & 78 & 22 & 5,1 & 0,9 & 28,4 & 2,5 & 991,1 & 258,4 & 9,7 & 4,2 \\
\hline
\end{tabular}

G1= ROP estágio II e III pós-tratamento; G2= ROP estágio II e III regredida; G3= sem ROP; M= masculino; F= feminino; ICR= idade cronológica na reavaliação; IG= idade gestacional; PN= peso de nascimento; $\mathrm{DP}=$ desvio padrão; $\mathrm{N}=$ número total 
Quadro 3. Características descritivas do exame de tomografia de coerência óptica (OCT) de pré-escolares nascidos prematuros componentes dos grupos G1, G2 e G3 em relação à espessura foveal e a características do vítreo e da interface vitreorretiniana

\begin{tabular}{lllccc}
\hline Grupos & OCT & N & Olho direito & N & Olho esquerdo \\
\hline G1 & Espessura foveal (micra) & 7 & $271(226-313)$ & $5^{*}$ & $257,8(217-280)$ \\
& Vítreo & 7 & S/ALT & $5^{*}$ & S/ALT \\
& Interface vitreorretiniana & 7 & S/ALT & $5^{*}$ & S/ALT \\
\multirow{2}{*}{ G2 } & Espessura foveal (micra) & 8 & $214,2(196-231)$ & 8 & $213,8(191-237)$ \\
& Vítreo & 8 & S/ALT & 8 & S/ALT \\
& Interface vitreorretiniana & 8 & S/ALT & 8 & S/ALT \\
\multirow{2}{*}{ G3 } & Espessura foveal (micra) & 9 & $219,3(176-258)$ & 9 & S/ALT \\
& Vítreo & 9 & S/ALT & 9 & S/ALT \\
\hline
\end{tabular}

G1= ROP estágio II e III pós-tratamento; G2= ROP estágio II e III regredida; G3= sem ROP; S/ALT= sem alteração.

NOTA: $\left(^{*}\right)$ dois prematuros não realizaram o exame no olho esquerdo, um por apresentar descolamento tardio de retina e outro por não permitir o exame neste olho.

Quadro 4. Características descritivas do exame de tomografia de coerência óptica $(O C T)$ de pré-escolares nascidos prematuros componentes dos grupos G1, G2 e G3 em relação às alterações retinianas

\begin{tabular}{|c|c|c|c|c|}
\hline Grupos & $\begin{array}{c}\text { Alterações } \\
\text { retinianas } \\
\text { ao OCT }\end{array}$ & $\begin{array}{l}\text { Pré-escolar } \\
\text { nascido } \\
\text { prematuro }\end{array}$ & Olho direito & Olho esquerdo \\
\hline \multirow[t]{7}{*}{ G1 } & & 1 & CFA e ERM & CFA \\
\hline & & 2 & RCF & RCF \\
\hline & & 3 & RCF e AERF & RCF e AERF \\
\hline & & 4 & CFA, AERF e ARSN & CFA e ARSN \\
\hline & & 5 & CFA, ERM e ARCIR & CFA, ERM e ARCIR \\
\hline & & 6 & RCF, ERM e ARCIR & NR \\
\hline & & 7 & RCF e ERM & $N R$ \\
\hline \multirow[t]{8}{*}{ G2 } & & 8 & CFP & CFP \\
\hline & & 9 & CFP e AECPEX & CFP \\
\hline & & 10 & CFA & CFP \\
\hline & & 11 & CFP & CFA e ERM \\
\hline & & 12 & CFP & CFP \\
\hline & & 13 & CFA & CFA \\
\hline & & 14 & CFP e ARCIR & CFP e ARCIR \\
\hline & & 15 & CFP & CFP \\
\hline \multirow[t]{9}{*}{ G3 } & & 16 & CFP & CFP \\
\hline & & 17 & CFP & CFP \\
\hline & & 18 & CFA, ERM & CFA, ERM \\
\hline & & 19 & CFA & CFA \\
\hline & & 20 & RCF, ERM & RCF, ERM \\
\hline & & 21 & CFA & CFA \\
\hline & & 22 & CFA & CFA \\
\hline & & 23 & CFP, ARCIR & CFP, ARCIR \\
\hline & & 24 & CFA & CFA \\
\hline
\end{tabular}

AERF=aumento da espessura da região foveal; $A E C P E X=$ aumento da espessura da camada plexiforme externa da retina; $A R C I R=$ aumento da refletividade da camada interna da retina; $A R S N=$ aumento da refletividade do setor nasal da retina; $C F A=$ contorno foveal atenuado (ainda mantem algum vestígio de depressão foveal); CFP= contorno foveal preservado; $E R M=$ espessamento da região macular; $N R=$ não realizado; $R C F=$ retificação do contorno foveal (sem nenhum vestígio de depressão foveal)
Tabela 1. Média comparativa da espessura foveal obtida pelo exame de tomografia de coerência optica (OCT) em pré-escolares nascidos prematuros componentes dos grupos $\mathrm{G} 1$ e $\mathrm{G} 2$ e entre os componentes dos grupos G1 e G3

\begin{tabular}{|c|c|c|c|c|}
\hline \multirow[b]{3}{*}{ Grupos } & \multicolumn{4}{|c|}{ Espessura foveal ao OCT } \\
\hline & \multicolumn{2}{|c|}{ Olho direito } & \multicolumn{2}{|c|}{ Olho esquerdo } \\
\hline & G1 & G2 & G1 & G2 \\
\hline N & 7 & 8 & 5 & 8 \\
\hline Média & 271,0 & 214,3 & 257,8 & 213,9 \\
\hline Mediana & 276,0 & 211,0 & 262,0 & 213,0 \\
\hline Mínimo & 226,0 & 196,0 & 217,0 & 191,0 \\
\hline Máximo & 313,0 & 231,0 & 280,0 & 237,0 \\
\hline DP & 29,5 & 11,9 & 24,7 & 15,4 \\
\hline Valor de $\mathrm{p}$ & \multicolumn{2}{|c|}{0,001} & \multicolumn{2}{|c|}{0,011} \\
\hline Grupos & G1 & G3 & G1 & G3 \\
\hline N & 7 & 9 & 5 & 9 \\
\hline Média & 271,0 & 219,3 & 257,8 & 223,4 \\
\hline Mediana & 276,0 & 224,0 & 262,0 & 225,0 \\
\hline Mínimo & 226,0 & 176,0 & 217,0 & 180,0 \\
\hline Máximo & 313,0 & 258,0 & 280,0 & 265,0 \\
\hline DP & 29,5 & 24,9 & 24,7 & 26,9 \\
\hline Valor de $p$ & \multicolumn{2}{|c|}{0,003} & \multicolumn{2}{|c|}{0,060} \\
\hline
\end{tabular}

p* Teste não paramétrico de Mann-Whitney, $\mathrm{p}<0,05$, para intervalo de confiança de $95 \%$. $\mathrm{G} 1=$ prematuros com ROP pós tratamento; $\mathrm{G} 2=$ prematuros com ROP e regressão espontânea; $\mathrm{G} 3=$ prematuros sem ROP.

grupo G1 principalmente, também prejudicou esta comparação. A comparação estatística entre os grupos G2 e G3 não foi realizada, desconsiderou-se esta necessidade diante do resultado obtido com a análise dos resultados da OCT entre G1 e G3.

Outros pesquisadores que realizaram uma pesquisa semelhante não encontraram diferença em relação à espessura foveal entre préescolares nascidos prematuros com ROP pós-tratamento e com ROP regredida e entre os nascidos prematuros com ROP e aqueles sem $\mathrm{ROP}^{(6)}$.

Apesar de a frequência de alterações nas camadas da retina entre os prematuros tratados para a regressão da retinopatia ter sido alta (100\%), não houve diferença estatisticamente significativa entre os grupos estudados. Em relação às alterações das camadas retinianas, os 


\begin{tabular}{|c|c|c|c|c|}
\hline \multirow[b]{2}{*}{ Grupo G1 e Grupo G2 } & \multicolumn{2}{|c|}{ Olho direito } & \multicolumn{2}{|c|}{ Olho esquerdo } \\
\hline & G1 & G2 & G1 & G2 \\
\hline \multirow[t]{2}{*}{ Sem alteração retiniana } & 0 & 4 & 0 & 5 \\
\hline & $0,00 \%$ & $50,00 \%$ & $0,00 \%$ & $62,50 \%$ \\
\hline \multirow[t]{2}{*}{ Com alteração retiniana } & 7 & 4 & 5 & 3 \\
\hline & $100,00 \%$ & $50,00 \%$ & $100,00 \%$ & $37,50 \%$ \\
\hline Total & 7 & 8 & 5 & 237,0 \\
\hline Valor de $\mathrm{p}$ & \multicolumn{2}{|c|}{0,077} & \multicolumn{2}{|c|}{0,075} \\
\hline Grupo G1 e Grupo G3 & G1 & G3 & G1 & G3 \\
\hline \multirow[t]{2}{*}{ Sem alteração retiniana } & 0 & 2 & 0 & 2 \\
\hline & $0,00 \%$ & $22,22 \%$ & $0,00 \%$ & $22,22 \%$ \\
\hline \multirow[t]{2}{*}{ Com alteração retiniana } & 7 & 7 & 5 & 7 \\
\hline & $100,00 \%$ & $77,78 \%$ & $100,00 \%$ & $77,78 \%$ \\
\hline Total & 7 & 9 & 5 & 9 \\
\hline Valor de $p$ & \multicolumn{2}{|c|}{0,475} & \multicolumn{2}{|c|}{0,475} \\
\hline
\end{tabular}

p*Teste não paramétrico de Mann-Whitney, p<0,05, para intervalo de confiança de $95 \%$.

Tabela 3. Comparação entre as alterações retinianas observadas ao exame de oftalmoscopia binocular indireta $(\mathrm{OBI})$ em pré-escolares nascidos prematuros componentes dos grupos $\mathrm{G} 1$ e $\mathrm{G} 2$ e entre os componentes dos grupos $\mathbf{G} 1$ e G3

\begin{tabular}{|c|c|c|c|c|}
\hline \multirow[b]{3}{*}{ Variável } & \multirow{2}{*}{\multicolumn{2}{|c|}{$\begin{array}{c}\text { Olho direito } \\
\text { Grupos }\end{array}$}} & \multirow{2}{*}{\multicolumn{2}{|c|}{$\begin{array}{c}\text { Olho esquerdo } \\
\text { Grupos }\end{array}$}} \\
\hline & & & & \\
\hline & G1 & G2 & G1 & G2 \\
\hline \multirow[t]{2}{*}{ Sem alteração retiniana à OBI } & 0 & 6 & 0 & 6 \\
\hline & $0,00 \%$ & $75,00 \%$ & $0,00 \%$ & $75,00 \%$ \\
\hline \multirow[t]{2}{*}{ Com alteração retiniana à OBI } & 7 & 2 & 7 & 2 \\
\hline & $100,00 \%$ & $25,00 \%$ & $100,00 \%$ & $25,00 \%$ \\
\hline
\end{tabular}

$\begin{array}{lllll}\text { Total } & 7 & 8 & 7 & 8\end{array}$

Valor de p* p: 0,007 p:007

Valor de $p<0,05$ para intervalo de confiança de $95 \%$.

Com alteração retiniana no grupo $\mathrm{G} 1=$ marcas de ablação deixadas pelo tratamento da ROP; Com alteração retiniana no grupo $\mathrm{G} 2=$ alterações não provocadas pelo tratamento para a ROP.

prematuros sem ROP foram mais comparáveis com o grupo tratado do que os prematuros com ROP regredida espontaneamente (Tabela 2). Uma explicação plausível é suportada por outros investigadores, de que estas alterações retinianas poderiam ser um processo inerente à prematuridade $\mathrm{e}^{(6,7)}$.

Na análise comparativa dos achados da oftalmoscopia binocular indireta entre os grupos G1 e G2, encontrou-se diferença estatisticamente significativa para o grupo $\mathrm{G} 1$ ( $p=0,007 \mathrm{AO}$, intervalo de confiança 95\%). No grupo G1 este resultado expressa as alterações secundárias às sequelas deixadas pelo tratamento na fase aguda da ROP, a laser ou por crioterapia. Todos os prematuros sem ROP apresentaram à $\mathrm{OB}$, retina aplicada com aspecto anatômico normal.

Todos os pacientes componentes dos três grupos realizaram o exame de OBI na idade pré-escolar (reavaliação), exceto o prematuro que apresentou descolamento tardio de retina (OE), no grupo G1. Todos apresentaram a região macular e foveal com brilho, depressão e pigmentação normais, resultado inesperado para os Grupos G1 e G2. Acreditava-se que naqueles pacientes do grupo G1 em que as cicatrizes do tratamento que tivessem se aproximado mais da região macular pudessem apresentar, em associação, um deslocamento da região foveal ou mesmo um desarranjo da arquitetura vascular.

Em relação às particularidades do grupo G2, também seria esperado que após a regressão espontânea da ROP, alguns casos apresentassem mudança da relação papilo-foveal ou alterações periféricas típicas da ROP cicatricial, principalmente em crianças prematuras que chegaram a ter uma classificação de ROP próxima ao critério exigido para indicação do tratamento(14,15). No grupo G2, um prematuro apresentou ROP estágio III plus, na extensão de quatro horas contínuas no OD e estágio III plus, oito horas descontínuas no OE, sem informação sobre a zona acometida; critérios que indicam o tratamento para a ROP. Por dificuldades técnicas, o tratamento a laser não foi realizado e o exame de revisão relevou, à $O B I$, a regressão espontânea da ROP, com preservação do polo posterior e sem sequela.

A grande maioria destes prematuros com doença plus foi selecionada para tratamento, segundo os critérios dos Comitês de 1984 e 1987 $7^{(13,14)}$. Entre 2003 e 2005, foram realizadas revisões da Classificação Internacional da ROP, e, em 2007 no Brasil, com o objetivo de regular a importância da presença da doença plus como base para a indicação de tratamento da ROP na zona I e zona II, em qualquer estágio da retinopatia ${ }^{(16-18)}$. Atualmente, a presença da doença plus é indicação indiscutível para tratamento, no entanto, na época em que os pré-escolares nascidos prematuros tiveram suas primeiras avaliações, a doença plus esteve presente em seis dos oito pacientes do grupo G2. Portanto, alguns pacientes classificados como G2 tinham ficado próximo à classificação para o grupo de G1 tratamento. No grupo G2 apenas dois prematuros apresentaram alteração à oftalmoscopia binocular indireta nos dois olhos: um com aumento da tortuosidade vascular e outro com aumento da escavação do nervo óptico, ambos com a região macular e foveal preservada.

A preservação anatômica das regiões maculares e foveais à oftalmoscopia indireta após o tratamento da ROP observada na presente pesquisa, também é reportada por outros grupos ${ }^{(15,19,20)}$.

Quanto à avaliação comparativa do aspecto fundoscópico com as imagens do OCT, relatou-se um fator em comum indiferentemente da metodologia empregada, a dificuldade de se relacionar os achados do exame de oftalmoscopia indireta com o aspecto observado nos exames de OCT em relação à anatomia do polo posterior ${ }^{(6,7,11)}$. Isto difere do que é encontrado em trabalhos de seguimento de pacientes adultos com diabetes onde os autores conseguem estabelecer boa correlação entre a biomicroscopia de fundo e as alterações encontradas na OCT, chegando a sugerir o exame como fator preditivo para tratamento(21). Neste estudo, os pacientes diabéticos sem edema macular prévio à panfotocagulação, apresentaram aumento da espessura foveal ao longo do seguimento, embora sem repercussões funcionais (21). Devido à fisiopatologia semelhante entre as duas doenças retinianas (diabetes e ROP, ambas vasoproliferativas), esperar-se-ia respostas semelhantes nos exames. Na avaliação das crianças prematuras não haviam sido realizadas as OCT prévias ao tratamento, na fase aguda ou imediatamente após a fase aguda da ROP, dados que poderiam auxiliar na comparação destes dois tipos de retinopatia.

A realização dos exames de OCT em crianças apresenta grau de complexidade técnica relacionada à colaboração, principalmente em prematuros em qualquer idade, pois este exame exige imobilidade e adequada fixação do olhar para obtenção de imagens de boa qualidade. As crianças prematuras apresentam alta incidência de problemas neuropsicomotores (controle inadequado do movimento corporal, de cabeça, ou postura inadequada) e maior dificuldade de permanecerem imóveis durante realização do exame. Estas características limitam a empregabilidade do exame de OCT em crianças. Atualmente existe a comercialização de equipamentos portáteis de 
OCT, mais indicados para o exame de crianças prematuras internadas e com fase ativa de ROP, mas ainda são de comercialização restrita.

De acordo com as mais recentes publicações relacionadas ao desenvolvimento foveal no ser humano nascido a termo e prematuro, procurou-se entender o fato de $100 \%$ dos prematuros tratados apresentarem alteração na estrutura da fóvea às imagens do OCT (Figura 1). Isto sugere uma modificação na estrutura retiniana foveal destes pacientes. Há dificuldade em se diferenciar se estes achados do grupo G1 seriam decorrentes da gravidade da ROP, da própria condição da prematuridade em si, ou ainda, se seriam causadas pelos tratamentos a laser ou por crioterapia realizados nas áreas tratadas. Estudos que relacionam a formação normal da região foveal humana são escassos. Data de 1986 uma pesquisa sobre a análise histológica do desenvolvimento foveal humano, que demonstrou que a região foveal é identificável após 22 semanas de idade gestacional e que permanece imatura até o nascimento. A formação da depressão foveal se dá próximo à época do nascimento e continua até 15 meses de idade ${ }^{(22)}$. A imaturidade da região foveal pode ter seu ritmo normal de desenvolvimento alterado no nascimento prematuro por várias razões. Nos prematuros com ROP tratados, em especial, a intervenção a laser ou por crioterapia, é um fator de influência a ser considerado. $\mathrm{O}$ tratamento da ROP geralmente é realizado entre as 36a a 48a semanas de idade gestacional, época em que a região foveal estaria no auge do seu ajuste e especificação(15,22).

Estudos atuais realizados em prematuros com a utilização da tomografia de coerência óptica, aparelhos de domínio espectral e
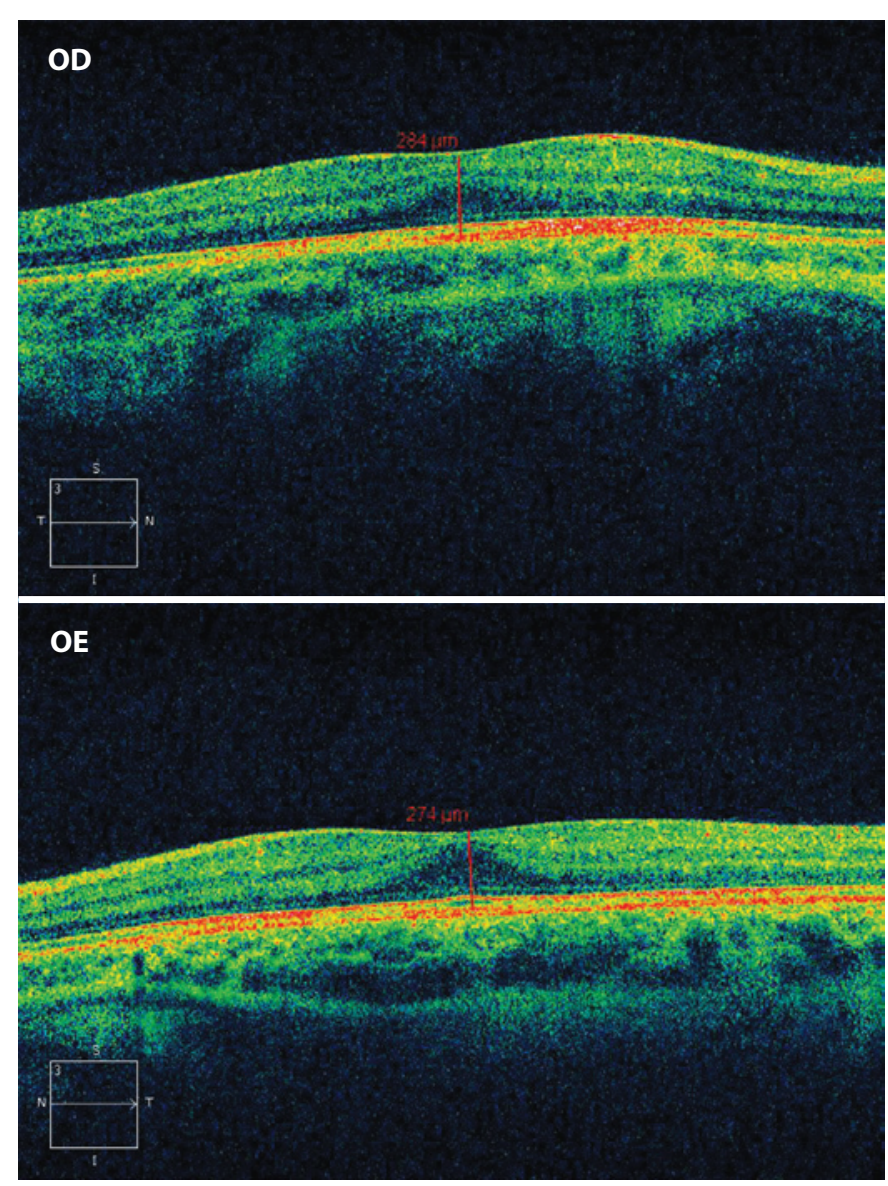

Figura 1. Tomografia de coerência óptica de olhos direito (OD) e esquerdo (OE) de pré-escolar do sexo masculino, aos 8 anos de idade, nascido prematuro, e componente do Grupo G1, com retinopatia da prematuridade regredida após tratamento a laser. Acuidade visual OD: 20/30; e OE: 20/25. portáteis, vem trazendo informações sobre a região macular e da retina destes prematuros ainda durante o período de seleção. Em 2007 foi publicado um estudo sobre tomografia de coerência óptica utilizada em 12 bebês prematuros com ROP (estágios 1, 2 e 3) e idade gestacional entre a 28a e 36a semanas, na época do exame. A depressão foveal esteve presente em 23\% destes prematuros ${ }^{(8)}$. Em 2011, um grupo publicou os resultado das imagens do OCT na fase aguda da ROP e, no mesmo grupo de pacientes, analisou as imagens do OCT após a resolução desta fase. Os autores descreveram dois tipos de alteração ao OCT durante a fase aguda da retinopatia da prematuridade: edema cistoide da mácula; e aumento moderado da espessura foveal e a depressão mantida, com associação a múltiplos vacúolos agrupados ou confluentes e opticamente hiporreflexivos localizados entre as camadas da retina. Os pesquisadores constataram que $100 \%$ destes olhos apresentavam o contorno foveal normalizado na segunda fase do estudo, independente do tipo de alteração encontrada durante a fase aguda da $\mathrm{ROP}^{(23)}$. Este dado obtido questiona a concepção de que as mudanças ocorridas na fase inicial da formação da fóvea do prematuro seriam mantidas até a vida adulta.

Em 2012, o OCT de domínio espectral foi avaliado em outro grupo de prematuros, que apresentavam entre 31 a 36 semanas de idade gestacional (para o calculo da IG, considerou-se o último período menstrual materno). Os autores se propuseram a observar se a severidade do edema cistoide de mácula, encontrado em 50\% dos 42 prematuros estudados, tinha algum valor preditivo para a necessidade de tratamento. Eles concluíram que o edema cistoide de mácula não estava diretamente relacionado com a gravidade preditiva para tratamento da retinopatia da prematuridade, mas a espessura foveal e parafoveal aumentada $\operatorname{sim}^{(24)}$. A relação encontrada neste estudo pode oferecer uma explicação para o que foi encontrado no grupo G1 do presente estudo, pois a espessura foveal foi significativamente maior nos prematuros deste grupo G1 em relação ao grupo G2. Infelizmente, a metodologia entre os estudos é variável, o que dificulta as comparações.

Por outro lado, é importante compreender o que acontece com as alterações observadas ao OCT na fase aguda, no momento da regressão após tratamento ou após sua regressão espontânea e, mais tarde, na infância e na vida adulta destes nascidos prematuros. Em 2010, autores encontraram alterações na arquitetura macular e foveal nas imagens do OCT em nascidos prematuros com ROP com idade média de 39 anos. As alterações encontradas foram: diminuição da depressão foveal, aumento da espessura macular e continuidade da camada interna da retina sobre a região foveal. Estes investigadores demonstraram com este estudo, que as alterações destes prematuros se mantêm até a vida adulta ${ }^{(7)}$. No entanto, estes são trabalhos isolados realizados em populações diferentes, com metodologia diversa. Não existe até o momento um trabalho linear, realizado com um mesmo grupo de prematuros, da fase aguda até fase a adulta, que unisse estes aspectos nos diferentes períodos de maturação foveal e retiniana do prematuro. A pesquisa ideal implicaria em complexidade metodológica e longo período de observação e de acompanhamento.

Neste trabalho, a amostra foi considerada homogênea nos três grupos em relação aos dados demográficos (idade no momento do re-exame, peso ao nascimento, idade gestacional). A dificuldade encontrada para reunir crianças de nascimento prematuro dentro dos critérios de inclusão prejudicou o tamanho da amostra, mas a alta incidência de alterações nas camadas da retina, observadas ao exame de $\mathrm{OCT}$, sugere que este seja relacionado à condição de prematuridade. $\mathrm{O}$ estudo não permitiu relacionar as alterações com a gravidade da ROP.

\section{CONCLUSÃO}

Os pré-escolares nascidos prematuros com ROP pós-tratamento apresentaram maior espessura foveal que os prematuros pós-re- 
gressão espontânea e apresentaram semelhança ao grupo de prematuros sem ROP, quando considerada a avaliação do olho esquerdo. As alterações nas camadas da retina da série de pacientes e observadas ao OCT não demonstraram diferença comparativa entre os três grupos do estudo.

O estudo sugere que o emprego da tecnologia de OCT em crianças nascidas prematuras pode contribuir para a compreensão das alterações estruturais relacionadas à condição de prematuridade.

\section{REFERÊNCIAS}

1. Simonsohn G, Kleeberger L, Plohn HJ. Die Verteilung des Brechungsindex in der Augenlinse. Optik. 1969;29:81-6.

2. Fercher AF. Optical coherence tomography - development, principles, applications. Z Med Phys. 2010;20(4):251-76. Review.

3. El-Dairi MA, Asrani SG, Enyedi LB, Freedmann SF. Optical coherence tomography in the eyes of normal children. Arch Ophthalmol. 2009;127(1):50-8.

4. Kee SY, Lee SY, Lee YC. Thicknesses of fovea and retinal nerve fiber layer in amblyopic and normal eyes in children. Korean J Ophthalmol. 2006;20(3):177-81.

5. Gupta G, Donahue JP, You T. Profile of the retina by optical coherence tomography in the pediatric age group. Am J Ophthalmol. 2007;144(2):309-10.

6. Ecsedy M, Szamosi A, Karkó C, Zubovics L, Varsányi B, Németh J, Récsán Z. A comparison of macular structure imaged by optical coherence tomography in preterm and full-term children. Invest Ophthalmol Vis Sci. 2007:48(11):5207-11.

7. Baker PS, Tasman W. Optical coherence tomography imaging of the fovea in retinopathy of prematurity. Ophthalmic Surg Lasers Imaging. 2010;41(2):201-6.

8. Lago Ad, Matieli L, Gomes M, Baba NT, Farah ME, Belfort Junior R, et al. Stratus optical coherence tomography findings in patients with retinopathy of prematurity. Arq Bras Oftalmol. 2007;70(1):19-21.

9. Soong GP, Shapiro M, Seiple W, Szlyk JP. Macular structure and vision of patients with macular heteropia secondary to retinopathy of prematurity. Retina. 2008;28(8): 1111-6.

10. Joshi MM, Trese MT, Capone A Jr. Optical coherence tomography findings in stage 4 A retinopathy of prematurity: a theory for visual variability. Ophthalmology. 2006; 113(4):657-60.

11. Recchia FM, Recchia CC. Foveal dysplasia evident by optical coherence tomography in patients with a history of retinopathy of prematurity. Retina. 2007;27(9):1221-6.

12. Lee AC, Maldonado RS, Sarin N, O'Connell RV, Wallace DK, Freedman SF, et al. Macu- lar features from spectral-domain optical coherence tomography as an adjunct to indirect ophthalmoscopy in retinopathy of prematurity. Retina. 2011;31(8):1470-82.

13. An international classification of retinopathy of prematurity. The Committee for the Classification of Retinopathy of Prematurity. Arch Ophthalmol. 1984;102(8):1130-4

14. An international classification of retinopathy of prematurity. II. The classification of retinal detachment. The International Committee for the Classification of the Late Stages of Retinopathy of Prematurity. Arch Ophthalmol. 1987;105(7):906-12. Erratum in Arch Ophthalmol. 1987;105(11):1498.

15. Katoch D, Sanghi G, Dogra MR, Beke N, Gupta A. Structural sequelae and refractive outcome 1 year after laser treatment for type 1 prethreshold retinopathy of prematurity in Asian Indian eyes. Indian J Ophthalmol. 2011;59(6):423-6.

16. International Committee for the Classification of Retinopathy of Prematurity. The International Classification of Retinopathy of Prematurity Revisited. Arch Ophthalmol. 2005;123(7):991-9.

17. Zin A, Florêncio T, Fortes Filho JB, Nakanami CR, Gianini N, Graziano RM, et al. Proposta de diretrizes brasileiras do exame e tratamento de retinopatia da prematuridade (ROP). Arq Bras Oftalmol. 2007;70(5):875-83.

18. Early Treatment for Retinopathy of Prematurity Cooperative Group. Revised indications for the treatment of retinopathy of prematurity: results of the early treatment for retinopathy of prematurity randomized trial. Arch Ophthalmol. 2003;121(12):1684-94. Comment in Arch Ophthalmol. 2005;123(3):406-7; discussion 409-10. Arch Ophthalmol. 2003;121(12):1769-71.

19. Talu S, Cormos D, Zaharia G, Stefanut C, Popa M, Lucaci DI. [Prognostic factors for laser treatment in retinopathy of prematurity]. Oftalmologia. 2011;55(1):84-9. Romanian.

20. Galván Ledesma A, Morales Guillén C, Pastor Ramos MT, Vázquez Marouschek Mdel C. [Diode laser photocoagulation of retinopathy of prematurity]. Arch Soc Esp Oftalmol. 2011;86(11):368-73. Spanish.

21. de Oliveira Maia Jr O, Takahasshi WY, Nakashima Y. Espessura macular e acuidade visual na retinopatia diabética tratada por panfotocoagulação. Arq Bras Oftalmol. 2008;71(3):394-9.

22. Yuodelis $C$, Hendrickson A. A qualitative and quantitative analysis of the human fovea during development. Vision Res. 1986;26(6):847-55.

23. Vinekar A, Avadhani K, Sivakumar M, Mahendradas P, Kurian M, Braganza S, et al Understanding clinically undetectal macular changes in early retinopathy of prematurity on spectral domain optical coherence tomography. Invest Ophthalmol Vis Sci. 2011;52(8):5183-8.

24. Maldonado RS, O'Connell R, Ascher SB, Sarin N, Freedman SF, Wallace DK, et al. Spectral-domain optical coherence tomographic assessment of severity of cystoid macular edema in retinopathy of prematurity. Arch Ophthalmol. 2012;130(5): 569-78. 